\title{
Online White Board
}

\author{
Prakhar Gupta ${ }^{1}$ Vasudha Bahl ${ }^{2}$
}

${ }^{1}$ B. Tech Scholar, Department of IT, Maharaja Agrasen Institute of Technology, Delhi, India

${ }^{2}$ Assistant Professor, Department of IT, Maharaja Agrasen Institute of Technology, Delhi, India

To Cite this Article

Prakhar Gupta and Vasudha Bahl, "Online White Board", International Journal for Modern Trends in Science and Technology, 6(12): 510-513, 2020.

\section{Article Info}

Received on 16-November-2020, Revised on 09-December-2020, Accepted on 12-December-2020, Published on 21-December-2020.

\section{ABSTRACT}

This paper focuses on a whiteboard project, which was made using web development which included both frontend basics such as HTML, CSS, JS, etc. and backend fundamentals which included but weren't limited to Mongo-DB, Node.JS, Express.JS etc. "ONLINE WHITEBOARD” is a real time application that allows user to draw/ write important points during a meeting and can also be used for teaching purposes while it helps the teacher to explain the topic in detail while taking the classes online. It has an intuitive interface that allows user to share ideas in real time. It also allows the user to save the current state of the browser for future purposes, which gets saves in users' local drive.

KEYWORDS: Whiteboard, online whiteboard, JavaScript, virtual whiteboard

\section{INTRODUCTION}

As COVID-19 is continuing to impact people around the world, thousands of schools and teachers are using Whiteboard as a key tool to provide the best remote learning experience and to help students stay engaged during remote learning.

With everyone staying and working from home these days, due to the COVID-19 lockdowns, the amount of online meetings and video conferences have skyrocketed. Many schools and design companies require systems that allow for collaborative sessions.

Major problem with the available online whiteboards is that most of the boards are locked behind a money vault or most of the features of the whiteboard are needed to be purchased, which makes it difficult for small organizations to afford the board. Hence making it difficult for virtual interactions and idea sharing.

Our whiteboard strives to solve these problems. Taking the magical simplicity of an analog whiteboard and adding interactive, collaborative technology, our Whiteboard gives the whole class a new space to engage, ideate, and create in real time. It will a free to use online whiteboard that anyone can use without paying huge sums of money.

\section{Scope Of The Project}

Whiteboard is designed specifically for the best online experience. It breaks past the physical limits of a traditional whiteboard and sprinkles in the brainstorm potential that a flexible cloud network empowers.Freehand drawing is possible and works great, but doesn't seem to be the largest focus. Whiteboard is designed for instant access and the ease of use, allowing users to jump online without logging in or downloading any apps, invite guests or coworkers, and quickly share ideas or draw visual explanations. With less of a focus on the 
longevity and direct replacement for office • whiteboards

- Add on post-it notes allow users to write down ideas or comments to spur collaboration and supply feedback without alerting the original idea - $\bullet$ definitely a solid function.

- Because the WhiteBoard is meant to be an infinite canvas, navigation is made easy with unique mouse controls

- Boards can also be saved as images, PDFs, downloaded as backups, saved to Google Drive

- Quick links make it super easy to invite anyone on your team, or even other guests, to jump in and collaborate. It should take less than 20 seconds to start a board, invite your team and have them jump right in.

- Share your experience whenever \& wherever you are. Distance and timing are no longer an obstacle.

- Make all students feel present. Let absent students participate as if they are in the classroom. Make classroom teaching easy and interactive via shared whiteboards.

- Invite external guests with a public link. Let parents and your future students get familiar with your knowledge and working style.

- Visualize teaching. Import JPEG, PNG images and PDF files to make your lessons easy to understand \& memorize.

- Share your knowledge and ideas with text and pen tool

- Make your work more visually enriched by uploading beautiful and topic-related images

- Enjoy infinite palette of colors and basic geometric figures like square, circle and ellipse

Export your boards when finished as PDF files Share the PDF files via email or publish them on soc. media as well as personal and professional blogs.

\section{What Makes A Great ONLINE WhiteBoARD?}

While any sketch or doodle app could technically be turned into a digital whiteboard, there are a few features that we consider integral to giving you the best digital whiteboarding experience:
Unlimited canvas. While physical whiteboards certainly have size restrictions, going digital should mean that you're no longer limited in how big or complex your visualizations are.

Collaboration features. Cloud apps should allow easy collaboration (real-time preferred) and comments by users. Desktop apps should allow adequate cloud file sharing/syncing across devices.

Ability to attach files: You should be able to attach links, images, and other files to your whiteboard for easy reference.

Presentation options. Because whiteboards are so often at the core of a collaborative process, you should be able to share your digital whiteboard online or export it.

\section{RELATED WORK}

A. Prasetya, Didik discussed in their paper the different limitations of e-learning such as a visual representation of diagrams and their explanations. To resolve these limitations they proposed a tool that connects students and teachers to a shared whiteboard. They have further discussed how their paper will help in distance learning and online collaborations.

B. Mâta, L., Lazar, G., \& Lazăr, I. in their research paper have discussed the impact of interactive whiteboards used in teaching and learning Science. They have researched the impact of interactive whiteboards in Learning Sciences and their findings reveal there are positive effects of the use of Interactive Whiteboards in learning and teaching Sciences. Pardanjac, Marjana \& Karuovic, Dijana \& Eleven, Erika in their paper has also discussed the possibilities of the use of interactive whiteboards and the use of this software in the teaching process.

C. Ringe, Swati \& Kedia, Rishabh \& Poddar, Anuj \& Patel, Sahil. discussed in their paper how HTML based whiteboard can be used in meeting collaborations and e-learning. They have used HTML5, Node.js \& Websockets to build their application. They have evaluated the performance of their application using New relic API and the statistics showcase a low response time. As the response time is low, users can see an instant change of data, and hence a high level of real-time communication is attained. 
D. Nikos Pinikas, Spyros Panagiotakis, Despina * Athanasaki, Athanasios G. Malamos discussed in their paper how high-quality real-time applications * can be developed by the use of WebRtc APIs. They have demonstrated certain use cases of WebRtc APIs in online collaboration such as screen recording, screen streaming, and sharing.

\section{TOOLS OF ONLINE WhiteBoARD}

Online whiteboard applications begin with an infinite canvas. This means there is no border to how big your whiteboard can be.

You can do freehand drawing with a number of pen tools like in Paint programs. The best whiteboard software can automatically straighten your lines. They can also recognize your shapes and transform them.

Widgets are very common tools of online whiteboards. These can be little icons that help guide the viewers. For example: arrows, sticky notes or post-it notes, stickers, highlights and more.

Notes are very important for online whiteboards. They allow team members to communicate with each other in exact places on the whiteboards. Notes can be saved as conversations or resolved.

You can also add media to online whiteboards. Most obvious are images and photos. But sometimes you can add video and audio tracks, or even upload other documents. These can be played or accessed by clicking directly inside the whiteboard.

May whiteboard platforms allow a variety of plugins and integrations too for added * functionality. These could be to publish whiteboards to web pages like WordPress, or to * include voice and video chatting with apps like * Skype.

\section{Methdology}

* The code for the whiteboard was written using the Visual Studio Code.

* Languages used for writing the code:

$>$ HTML - To provide structure

$>$ JavaScript - To provide functionality

$>\mathrm{CSS}$ - to provide design

* Node.js was used as an asynchronous event driven JavaScript runtime.
Canvas API had been used in the whiteboard to draw on the blank canvas.

Npm was integrated with Visual Studio Code.

Event Listeners were added for the user to draw on board.

Express will be used to provide server.

\section{Conclusion}

Online Whiteboard is an application that allows user to draw/ write important points during a meeting and can also be used for teaching purposes while it helps the teacher to explain the topic in detail while taking the classes online. It has an intuitive interface that allows users to communicate in real-time. It also allows the user to save the current state of the browser for future purposes, which gets saves in user's local drive. It can be used in meeting and corporate discussions to provide a streamlined work management. The whiteboard can be used in development cycles in firms to share and collaborate, to make mind maps and increase efficiency.

The uses of an online whiteboard are as limited as your imagination.

\section{Future Scope AND Possible UPdates}

Our whiteboard possess a great scope for the future:

Real time sharing could be added

* Online chatting could be added during presentation

Direct Integration with social networking and virtual meeting apps could be provided

Video calling could be added

Saving the board on Google drive

* Creating cloud storage for the board

* Various different pages could be added

- Virtual assistants could be added in the board

- Links can be created for easy sharing

* Various people can work on the same board together

* Security could be enhanced with login requirement

* Code editor could be integrated to help programmers and teachers.

These features could just be a start of a revolutionary Online Whiteboard. 


\section{REFERENCES}

[1] Prasetya Didik Dwi, and Muhammad Ashar. "Design of Interactive Whiteboard to Support E-Learning." www.Atlantis-Press.com, Atlantis Press, 1 Nov. 2017, www.atlantis-press.com/proceedings/icovet-17/2588641 2 .

[2] Mâță, Liliana, et al. "INTERACTIVE WHITEBOARDS FOR TEACHING AND LEARNING SCIENCE: ASCERTAINED RESEARCH." Journal of Innovation in Psychology, Education and Didactics, vol. 20, no. 2,2016,pp.135-148,files.eric.ed.gov/fulltext/ED572335.p df.

[3] Ringe, Swati \& Kedia, Rishabh \& Poddar, Anuj \& Patel, Sahil. (2015). HTML5 Based Virtual Whiteboard for Real Time Interaction. Procedia Computer Science. 49. 170-177. 10.1016/j.procs.2015.04.241.

[4] Pinikas, Nikos. (2017). A Device Independent Platform for Synchronous Internet of Things Collaboration and Mobile Devices Screen Casting. International Journal of Information and Communication Sciences. 2. 10.11648/j.ijics.20170205.12.

[5] Lant, Carol Le, and Michael J. Lawson. "Interactive Whiteboard Use and Student Engagement." Publishing Higher Degree Research, 2016, pp. 33-42, 10.1007/978-94-6300-672-9_4.

[6] Pardanjac, Marjana \& Karuovic, Dijana \& Eleven, Erika. (2018). The Interactive Whiteboard and Educational Software as an Addition to the Teaching Process. Tehnicki Vjesnik. 25. 255-262. 10.17559/TV-20160310173155.

[7]https://conceptboard.com/blog/virtual-whiteboard-for-be tter-teamwork-and-brainstorming/

[8]https://www.collaboard.app/en/blog/what-is-an-onlinewhi teboard-and-how-to-use-it-in-your-daily-work

[9]https://developer.mozilla.org/en-US/docs/Web/API/Canva s_API

[10]https://developer.mozilla.org/en-US/docs/Web/JavaScrip t/Reference/Statements/async_function

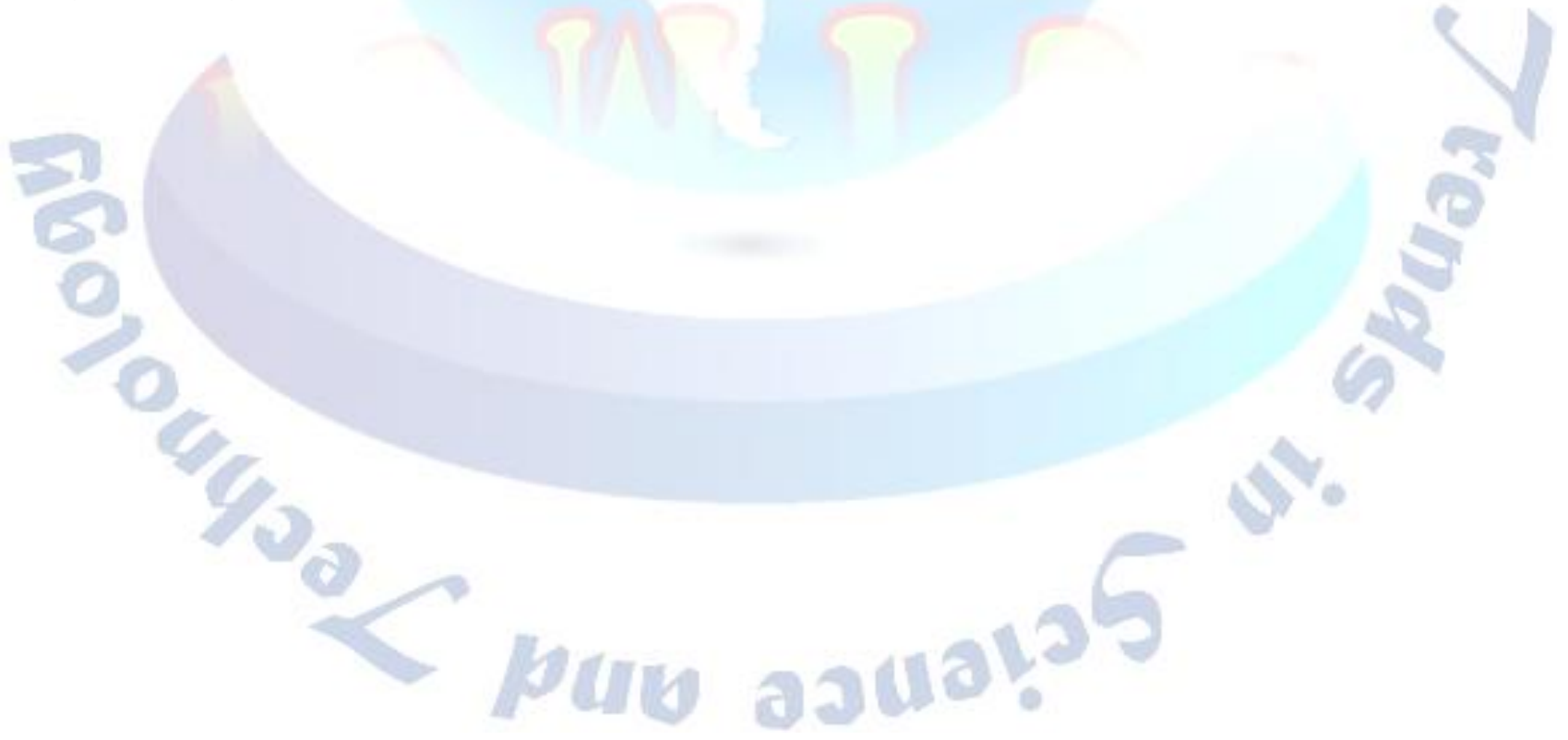

